\title{
Large Time Behavior for Weak Solutions of the 3D Globally Modified Navier-Stokes Equations
}

\author{
Junbai Ren \\ Research Center of Applied Statistics, School of Statistics, Jiangxi University of Finance and Economics, Nanchang 330013, China \\ Correspondence should be addressed to Junbai Ren; junbai_ren@jxufe.edu.cn
}

Received 28 April 2014; Accepted 2 June 2014; Published 12 June 2014

Academic Editor: Bo-Qing Dong

Copyright (C) 2014 Junbai Ren. This is an open access article distributed under the Creative Commons Attribution License, which permits unrestricted use, distribution, and reproduction in any medium, provided the original work is properly cited.

\begin{abstract}
This paper is concerned with the large time behavior of the weak solutions for three-dimensional globally modified Navier-Stokes equations. With the aid of energy methods and auxiliary decay estimates together with $L^{p}-L^{q}$ estimates of heat semigroup, we derive the optimal upper and lower decay estimates of the weak solutions for the globally modified Navier-Stokes equations as $C_{1}(1+t)^{-3 / 4} \leq\|u\|_{L^{2}} \leq C_{2}(1+t)^{-3 / 4}, t>1$. The decay rate is optimal since it coincides with that of heat equation.
\end{abstract}

\section{Introduction}

It is well known that the motion of the viscous incompressible fluids is governed by the following classic Navier-Stokes equations [1]:

$$
\begin{gathered}
\partial_{t} u+(u \cdot \nabla u)-\Delta u+\nabla p=0, \\
\nabla \cdot u=0 .
\end{gathered}
$$

Here $u$ and $\pi$ denote the unknown velocity and pressure of the fluid motion, respectively. This motion essentially presumes that the derivatives of the components of the velocity are small.

In Leray's pioneer work [2] in 1930, for any initial data in $L^{2}$, Navier-Stokes equations (1) exits a global weak solution $u$ satisfying

$$
u \in L^{\infty}\left(0, T ; L^{2}\right) \cap L^{2}\left(0, T ; H^{1}\right), \quad \text { for any } T>0 .
$$

However, the question of global existence for smooth solutions of the 3D Navier-Stokes equations is still a big open problem. In order to overcome this large difficulty, many efforts have been made to study some related modified Navier-Stokes equations (see [3, 4]). Recently, Caraballo et al. [5] (see also Kloeden et al. [6, 7]) introduced an interesting and important mathematical model which is the so-called global modification of the Navier-Stokes equations

$$
\begin{gathered}
\partial_{t} u+F_{N}\left(\|\nabla u\|_{L^{2}}\right)(u \cdot \nabla u)-v \Delta u+\nabla p=0, \\
\nabla \cdot u=0
\end{gathered}
$$

associated with

$$
u(x, 0)=u_{0},
$$

where $F_{N}$ (for some $N \in \mathbf{R}^{+}$) is defined by

$$
F_{N}(r)=\min \left\{1, \frac{N}{r}\right\}, \quad r \in \mathbf{R}^{+} .
$$

Let us give a profile analysis to this globally defined model. The modifying factor $F_{N}\left(\|\nabla u\|_{L^{2}}\right)$ is a function of $\|\nabla u\|_{L^{2}}$. Essentially, it prevents large gradients dominating flux and leading to explosions. What is the most important is that this model exhibits a unique global weak solution for the system (1) in bounded domain (see [5]).

However, it should be mentioned that although the presence of $F_{N}\left(\|\nabla u\|_{L^{2}}\right)$ actually canceled some singularities of the nonlinear term $u \cdot \nabla u$, it cannot increase the effect of low frequency of the solutions of the system (3). Therefore, it is interesting to consider the time decay issue of this model which largely depended on the effect of low frequency of the solutions. In this paper, we are focused on the $L^{2}$ decay 
of weak solutions for the modified Navier-Stokes equations (3). To carry out this issue, it is necessary to recall some classic time decay results of the fluid dynamical models. $L^{2}$ decay of weak solutions for the Navier-Stokes equations was first studied by Schonbek [8] (see also [9]). She first posed interesting methods the so-called Fourier splitting methods and the finite energy weak solutions decay as

$$
c(1+t)^{-n / 4} \leq\|u(t)\|_{L^{2}} \leq c_{1}(1+t)^{-n / 4} .
$$

Later on there are large good results to develop the Fourier splitting methods on the incompressible Navier-Stokes equations [10]. One may also refer to some interesting decay issues of the related fluid models [11-13].

Motivated by the upper and lower decay estimates of nonlinear fluid models [14], in this study, we will develop another technique to deal with the time decay problem of the weak solutions for the globally modified Navier-Stokes equations (3). Our trick is mainly based on the energy methods together with the $L^{p}-L^{q}$ estimates of heat semigroup in whole space $\mathbf{R}^{3}$. We can get the optimal time decay rate, since it coincides with that of linear equations.

\section{Preliminaries and Main Result}

In this paper, we denote by $C$ a generic positive constant which may vary from line to line.

$L^{p}\left(\mathbf{R}^{3}\right)$ with $1 \leq p \leq \infty$ is denoted by the Lebesgue space associated with the norm

$$
\|g\|_{L^{q}}= \begin{cases}\left(\int_{\mathbf{R}^{3}}|g(x)|^{q} d x\right)^{1 / q}, & 1 \leq q<\infty, \\ \operatorname{ess} \sup _{x \in \mathbf{R}^{3}}|g(x)|, & q=\infty .\end{cases}
$$

$H^{s}\left(\mathbf{R}^{3}\right)$ with $s \in \mathbf{R}$ is denoted by the fractional Sobolev space with the norm

$$
\|g\|_{H^{s}}=\left(\int_{\mathbf{R}^{3}}|\xi|^{2 s}|\hat{g}|^{2} d \xi\right)^{1 / 2}
$$

where $\hat{g}$ is the Fourier transformation

$$
\mathscr{F} g(\xi)=\widehat{g}(\xi)=\int_{\mathbf{R}^{3}} e^{-i x \cdot \xi} g(x) d x .
$$

$L^{q}(0, T ; X)$ is the space of all measurable functions $u$ : $(0, T) \mapsto X$ with the norm

$$
\|u\|_{L^{q}(0, T ; X)}=\left(\int_{0}^{T}\|u\|_{X}^{q} d t\right)^{1 / q}, \quad 1 \leq q<\infty,
$$

and when $q=\infty$,

$$
\|u\|_{L^{\infty}(0, T ; X)}=\operatorname{ess} \sup _{t \in[0, T]}\|u\|_{X} .
$$

To state the main results of this paper, we first give the definition of the weak solutions of the three-dimensional globally modified Navier-Stokes equations (3) [5].
Definition 1. $u(x, t)$ is called a weak solution for threedimensional globally modified Navier-Stokes equations (3) associated with $u_{0} \in L^{2}\left(\mathbf{R}^{3}\right)$ if the following properties

(i) $u \in L^{\infty}\left(0, T ; L^{2}\left(\mathbf{R}^{3}\right)\right) \cap L^{2}\left(0, T ; H^{1}\left(\mathbf{R}^{3}\right)\right)$;

(ii) for any $\phi \in C_{0}^{\infty}\left(\mathbf{R}^{3} \times[0, T)\right)$ with $\nabla \cdot \phi=0$,

$$
\begin{aligned}
& \int_{0}^{T} \int_{\mathbf{R}^{3}}\left\{u \cdot \partial_{t} \phi-\nabla u \cdot \nabla \phi+F_{N}\left(\|\nabla u\|_{L^{2}}\right) \nabla \phi: u \otimes u\right\} d x d t \\
& =-\int_{\mathbf{R}^{3}} u_{0} \phi(0) d x
\end{aligned}
$$

(iii) the energy inequality

$$
\begin{array}{r}
\|u(t)\|_{L^{2}}^{2}+2 \int_{0}^{t} \int_{\mathbf{R}^{3}}|\nabla u(x, s)|^{2} d x d s \leq\left\|u_{0}\right\|_{L^{2}}^{2}, \\
\text { for } 0 \leq t \leq T
\end{array}
$$

hold true.

Our results read as follows.

Theorem 2. Suppose that $u(x, t)$ is a weak solution for threedimensional globally modified Navier-Stokes equations (3). Moreover, if the solution $e^{\Delta t} u_{0}$ of the heat equation $\partial_{t} u-\Delta u=0$ satisfies

$$
C_{1}(1+t)^{-3 / 4} \leq\left\|e^{\Delta t} u_{0}\right\|_{L^{2}} \leq C_{2}(1+t)^{-3 / 4},
$$

then the weak solution $u(x, t)$ of (1) possesses the following optimal upper and lower decay rate:

$$
C_{1}(1+t)^{-3 / 4} \leq\|u\|_{L^{2}} \leq C_{2}(1+t)^{-3 / 4}, \quad t>1 .
$$

Remark 3. The decay rate is optimal since it coincides with that of heat equation. The finding is mainly based on energy methods and auxiliary decay estimates together with $L^{p}-L^{q}$ estimates of heat semigroup.

\section{Auxiliary $L^{2}$ Decay}

In this section, we will first study auxiliary $L^{2}$ decay of weak solutions for three-dimensional globally modified NavierStokes equations (3).

Lemma 4. Suppose that $u(t)$ is a weak solution of threedimensional globally modified Navier-Stokes equations (3); then one has

$$
t^{1 / 2}\|\nabla u(t)\|_{L^{2}} \longrightarrow 0, \quad t \longrightarrow \infty .
$$

Proof of Lemma 4. Taking the inner product of (3) with $-\Delta u$ gives

$$
\begin{aligned}
& \frac{d}{d t}\|\nabla u(t)\|_{L^{2}}^{2}+2\|\Delta u(t)\|_{L^{2}}^{2} \\
& \quad \leq\left|2 \int_{\mathbf{R}^{3}} F_{N}\left(\|\nabla u\|_{L^{2}}\right)(u \cdot \nabla) u \Delta u d x\right|,
\end{aligned}
$$


where we have used the following properties:

$$
\int_{\mathbf{R}^{3}} \nabla p \Delta u d x=\int_{\mathbf{R}^{3}} p \Delta(\operatorname{div} u) d x=0
$$

due to the divergence free of the velocity fields.

Since

$$
F_{N}\left(\|\nabla u\|_{L^{2}}\right)=\min \left\{1, \frac{N}{\|\nabla u\|_{L^{2}}}\right\} \leq 1,
$$

then the right hand side of inequality (17) can be estimated after by applying Hölder inequality

$$
\begin{gathered}
\left|2 \int_{\mathbf{R}^{3}} F_{N}\left(\|\nabla u\|_{L^{2}}\right)(u \cdot \nabla) u \Delta u d x\right| \\
\leq C\|\Delta u\|_{L^{2}}\|u\|_{L^{\infty}}\|\nabla u\|_{L^{2}} .
\end{gathered}
$$

With the aid of the Gagliardo-Nirenberg inequality,

$$
\begin{gathered}
\|u\|_{L^{\infty}} \leq C\|\Delta u\|_{L^{2}}^{3 / 4}\|u\|_{L^{2}}^{1 / 4}, \\
\|\nabla u\|_{L^{2}} \leq C\|u\|_{L^{2}}^{1 / 2}\|\Delta u\|_{L^{2}}^{1 / 2} .
\end{gathered}
$$

Plugging (21) into (20), one shows that

$$
\begin{aligned}
& \left|2 \int_{\mathrm{R}^{3}} F_{N}\left(\|\nabla u\|_{L^{2}}\right)(u \cdot \nabla) u \Delta u d x\right| \\
& \quad \leq C\|\Delta u\|_{L^{2}}^{7 / 4}\|u\|_{L^{2}}^{1 / 4}\|\nabla u\|_{L^{2}} \\
& \quad \leq C\|\Delta u\|_{L^{2}}^{2}\left(\|u\|_{L^{2}}^{1 / 4}\|\nabla u\|_{L^{2}}\|\Delta u\|_{L^{2}}^{-1 / 4}\right) \\
& \quad \leq C\|\Delta u\|^{2}\left(\|u\|_{L^{2}}^{1 / 2}\|\nabla u\|_{L^{2}}^{1 / 2}\right) .
\end{aligned}
$$

Then inserting the above inequality into (17), one gets

$$
\begin{aligned}
& \frac{d}{d t}\|\nabla u(t)\|_{L^{2}}^{2}+2\|\Delta u(t)\|_{L^{2}}^{2} \\
& \quad \leq C\|\Delta u(t)\|_{L^{2}}^{2}\|u\|_{L^{2}}^{1 / 2}\|\nabla u\|_{L^{2}}^{1 / 2}
\end{aligned}
$$

Thus we rewrite inequality (23) as

$$
\frac{d}{d t}\|\nabla u(t)\|_{L^{2}}^{2} \leq 2\|\Delta u(t)\|_{L^{2}}^{2}\left(C\|u\|_{L^{2}}^{1 / 2}\|\nabla u\|_{L^{2}}^{1 / 2}-1\right) .
$$

Now for any small $\varepsilon>0$, there exists a large $M>0$, such that, for $t \geq M$,

$$
\|u\|_{L^{2}}\|\nabla u\|_{L^{2}} \leq \varepsilon
$$

Otherwise, there exists a positive constant $\varepsilon_{0}$, such that, for all $t \geq 0$

$$
\|u\|_{L^{2}}\|\nabla u\|_{L^{2}}>\varepsilon_{0}
$$

from which and together with energy inequality we have

$$
\left\|u_{0}\right\|_{L^{2}}\|\nabla u\|_{L^{2}}>\varepsilon_{0}
$$

which implies that

$$
\|\nabla u\|_{L^{2}}>C \varepsilon_{0} .
$$

On the other hand, from energy inequality we have

$$
\int_{0}^{\infty}\|\nabla u\|_{L^{2}} d t<\infty
$$

which contradicts (28).

Hence, we have

$$
\|u\|_{L^{2}}\|\nabla u\|_{L^{2}} \leq \varepsilon, \quad \text { for large } t \text {. }
$$

We now choose $\varepsilon=1 / C$ in (24) and apply (30) to yield

$$
\frac{d}{d t}\|\nabla u(t)\|_{L^{2}}^{2} \leq 0
$$

from which and together with the energy inequality we have

$$
\begin{aligned}
& (t-M)\|\nabla u(t)\|_{L^{2}}^{2} \\
& \quad \leq \int_{M}^{t}\|\nabla u(s)\|_{L^{2}}^{2} d s \leq \frac{1}{2}\|u(M)\|^{2} \leq \frac{1}{2}\left\|u_{0}\right\|^{2}=C
\end{aligned}
$$

which implies that

$$
t\|\nabla u(t)\|_{L^{2}}^{2} \longrightarrow 0, \quad t \longrightarrow \infty .
$$

\section{Optimal Upper and Lower Decay Estimates}

4.1. Upper Decay Estimate. Consider the integral equations of (3)

$$
u(t)=e^{\Delta t} u_{0}-\int_{0}^{t} e^{\Delta(t-s)} \mathscr{P} F_{N}\left(\|\nabla u\|_{L^{2}}\right)(u \cdot \nabla u) d s,
$$

where

$$
\mathscr{P} g=g-\nabla \Delta^{-1} \nabla \cdot g \text {. }
$$

Taking the $L^{2}$ norm of the integral equation and applying the $L^{p}-L^{q}$ estimates of heat equation, it follows that together with Hölder inequality

$$
\begin{aligned}
& \|u(t)\|_{L^{2}} \\
& \leq\left\|e^{\Delta t} u_{0}\right\|_{L^{2}}+C \int_{0}^{t}\left\|e^{\Delta(t-s)} \mathscr{P} F_{N}\left(\|\nabla u\|_{L^{2}}\right)(u \cdot \nabla u)\right\|_{L^{2}} d s \\
& \leq C(1+t)^{-3 / 4}+C \int_{0}^{t}(t-s)^{-1 / 2}\|u\|_{L^{2}}\|\nabla u\|_{L^{2}} d s,
\end{aligned}
$$

where we have used the properties

$$
\begin{gathered}
\|\mathscr{P}\| \leq 1, \\
F_{N}\left(\|\nabla u\|_{L^{2}}\right) \leq 1 .
\end{gathered}
$$


According to Lemma 4, we let

$$
\eta(t) \triangleq t^{1 / 2}\|\nabla u(t)\|_{L^{2}}
$$

and it is obvious that

$$
\eta(t) \longrightarrow 0, \quad t \longrightarrow \infty .
$$

Thus we rewrite (36) as

$$
\begin{aligned}
& \|u(t)\|_{L^{2}} \\
& \leq\left\|e^{\Delta t} u_{0}\right\|_{L^{2}}+c \int_{0}^{t}\left\|e^{\Delta(t-s)} \mathscr{P} F_{N}\left(\|\nabla u\|_{L^{2}}\right)(u \cdot \nabla u)\right\|_{L^{2}} d s \\
& \leq C(1+t)^{-3 / 4}+C \int_{0}^{t}(t-s)^{-1 / 2}(1+s)^{-1 / 2}\|u\|_{L^{2}} \eta(s) d s .
\end{aligned}
$$

That is to say,

$$
(1+t)^{3 / 4}\|u(t)\|_{L^{2}} \leq C+C \theta(t) \sup _{0 \leq s \leq t}(1+s)^{3 / 4}\|u(s)\|_{L^{2}}
$$

with

$$
\theta(t)=(1+t)^{3 / 4} \int_{0}^{t}(t-s)^{-1 / 2}(1+s)^{-5 / 4} \eta(s) d s .
$$

It is easy to check that

$$
\theta(t) \longrightarrow 0, \quad \text { as } t \longrightarrow \infty,
$$

or for large $t>0$,

$$
\theta(t) \leq \frac{1}{2 C}
$$

Thus we obtain the optimal upper decay estimates of the weak solution for three-dimensional globally modified Navier-Stokes equations (3) as

$$
\|u(t)\|_{L^{2}} \leq C(1+t)^{-3 / 4}
$$

4.2. Lower Decay Estimate. From the integral equations (34), we will investigate the error estimates of solutions between three-dimensional globally modified Navier-Stokes equations (3) and the heat equation:

$$
\begin{aligned}
& \left\|u(t)-e^{\Delta t} u_{0}\right\|_{L^{2}} \\
& \leq \int_{0}^{t}\left\|e^{\Delta(t-s)} \mathscr{P} F_{N}\left(\|\nabla u\|_{L^{2}}\right)(u \cdot \nabla u)\right\|_{L^{2}} d s \\
& \leq \int_{0}^{t}\left\|e^{\Delta(t-s)}(u \cdot \nabla u)\right\|_{L^{2}} d s \\
& \leq \int_{0}^{t / 2}\left\|\nabla e^{\Delta(t-s)}(u \otimes u)\right\|_{L^{2}} d s \\
& \quad+\int_{t / 2}^{t}\left\|\nabla e^{\Delta(t-s)}(u \otimes u)\right\|_{L^{2}} d s \\
& =I+J .
\end{aligned}
$$

For $I$, employing the $L^{p}-L^{q}$ estimates of heat semigroup and upper decay estimates gives

$$
\begin{aligned}
I & \leq C \int_{0}^{t / 2}(t-s)^{-5 / 4}\|u\|_{L^{2}}^{2} d s \\
& \leq C \int_{0}^{t / 2}(t-s)^{-5 / 4}(1+s)^{-3 / 2} d s \\
& \leq C(1+t)^{-3 / 2} .
\end{aligned}
$$

For $J$, similarly,

$$
\begin{aligned}
J & \leq \int_{t / 2}^{t}\left\|\nabla e^{\Delta(t-s)}(u \otimes u)\right\|_{L^{2}} d s \\
& \leq \int_{t / 2}^{t}(t-s)^{-7 / 8}\|u \otimes u\|_{L^{4 / 3}} d s \\
& \leq \int_{t / 2}^{t}(t-s)^{-7 / 8}\|u\|_{L^{2}}\|u\|_{L^{4}} d s \\
& \leq \int_{t / 2}^{t}(t-s)^{-7 / 8}\|u\|_{L^{2}}^{5 / 4}\|\nabla u\|_{L^{2}}^{3 / 4} d s \\
& \leq \int_{t / 2}^{t}(t-s)^{-7 / 8}(1+s)^{-21 / 16} \eta^{3 / 4}(t) d s \\
& =o\left((1+t)^{-19 / 16}\right), \quad t \longrightarrow \infty .
\end{aligned}
$$

Thus we have from the estimates $I$ and $J$

$$
\left\|u(t)-e^{\Delta t} u_{0}\right\|_{L^{2}}=o\left((1+t)^{-19 / 16}\right), \quad t \longrightarrow \infty .
$$

Hence by the triangle inequality, one shows that

$$
\begin{aligned}
\|u(t)\|_{L^{2}} & \geq\left\|e^{-\Delta t} u_{0}\right\|_{L^{2}}-\left\|u(t)-e^{\Delta t} u_{0}\right\|_{L^{2}} \\
& \geq C(1+t)^{-3 / 4}, \quad \text { for large } t .
\end{aligned}
$$

Combination of the upper and lower decay estimates for weak solutions of three-dimensional globally modified Navier-Stokes equations (3) completes the proof of Theorem 2.

\section{Conflict of Interests}

The author declares that there is no conflict of interests regarding the publication of this paper.

\section{Acknowledgment}

This work is partially supported by the NSF of China nos. 11361026, 11161022, and 71161013.

\section{References}

[1] R. Teman, The Navier-Stokes Equations, North-Holland, Amsterdam, The Netherlands, 1977.

[2] J. Leray, "Sur le mouvement d'un liquide visqueux emplissant l'espace," Acta Mathematica, vol. 63, no. 1, pp. 193-248, 1934. 
[3] L. Caffarelli, R. Kohn, and L. Nirenberg, "Partial regularity of suitable weak solutions of the Navier-Stokes equations," Communications on Pure and Applied Mathematics, vol. 35, no. 6, pp. 771-831, 1982.

[4] B.-Q. Dong and Z. Zhang, "On the weak-strong uniqueness of Koch-Tataru's solution for the Navier-Stokes equations," Journal of Differential Equations, vol. 256, no. 7, pp. 2406-2422, 2014.

[5] T. Caraballo, J. Real, and P. E. Kloeden, "Unique strong solutions and V-attractors of a three dimensional system of globally modified Navier-Stokes equations," Advanced Nonlinear Studies, vol. 6, pp. 411-436, 2006.

[6] T. Caraballo, P. E. Kloeden, and J. Real, "Invariant measures and statistical solutions of the globally modified navier-stokes equations," Discrete and Continuous Dynamical Systems B, vol. 10, no. 4, pp. 761-781, 2008.

[7] P. E. Kloeden, J. A. Langa, and J. Real, "Pullback V-attractors of the 3-dimensional globally modified Navier-Stokes equations," Communications on Pure and Applied Analysis, vol. 6, no. 4, pp. 937-955, 2007.

[8] M. E. Schonbek, " $L^{2}$ decay for weak solutions of the NavierStokes equations," Archive for Rational Mechanics and Analysis, vol. 88, no. 3, pp. 209-222, 1985.

[9] R. Kajikiya and T. Miyakawa, "On $L^{2}$ decay of weak solutions of the Navier-Stokes equations in $\mathbb{R}^{n}$," Mathematische Zeitschrift, vol. 192, no. 1, pp. 135-148, 1986.

[10] L. Zhang, "New results of general $n$-incompressible NavierStokes equations," Journal of Differential Equations, vol. 245, no. 11, pp. 3470-3502, 2008.

[11] M. Dai, J. Qing, and M. Schonbek, "Asymptotic Behavior of Solutions to Liquid Crystal Systems in $\mathbb{R}^{3}$," Communications in Partial Differential Equations, vol. 37, no. 12, pp. 2138-2164, 2012.

[12] Y. Jia, X. Zhang, and B.-Q. Dong, "The asymptotic behavior of solutions to three-dimensional NavierStokes equations with nonlinear damping," Nonlinear Analysis: Real World Applications, vol. 12, no. 3, pp. 1736-1747, 2011.

[13] B. Q. Dong and Q. Q. Liu, "Asymptotic profile of solutions to the two-dimensional dissipative quasi-geostrophic equation," Science China Mathematics, vol. 53, no. 10, pp. 2733-2748, 2010.

[14] B. Dong and W. Jiang, "On the decay of higher order derivatives of solutions to Ladyzhenskaya model for incompressible viscous flows," Science in China A: Mathematics, vol. 51, no. 5, pp. 925934, 2008. 


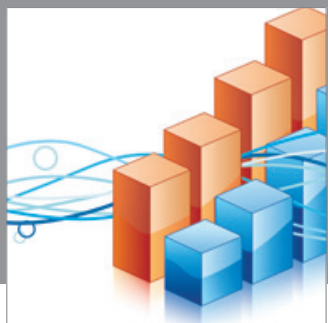

Advances in

Operations Research

mansans

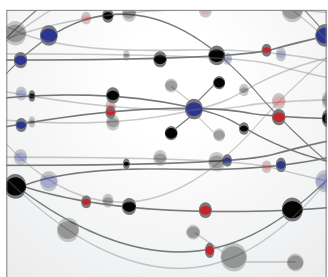

The Scientific World Journal
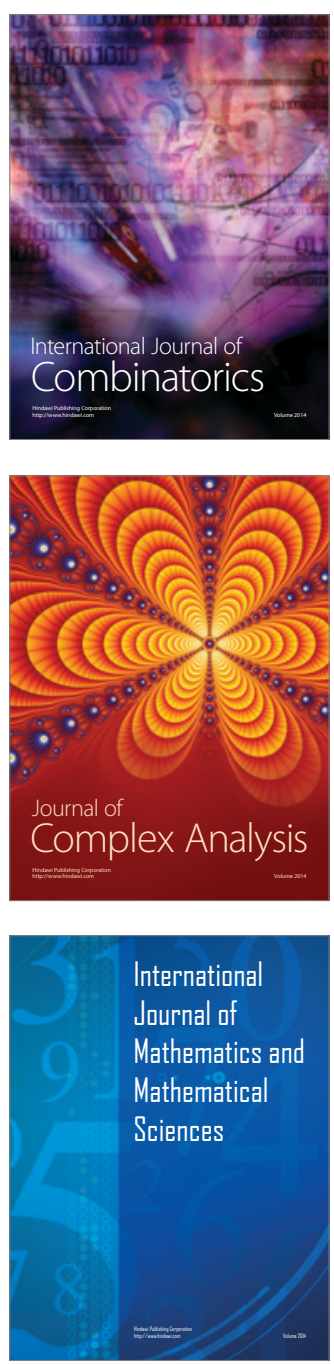
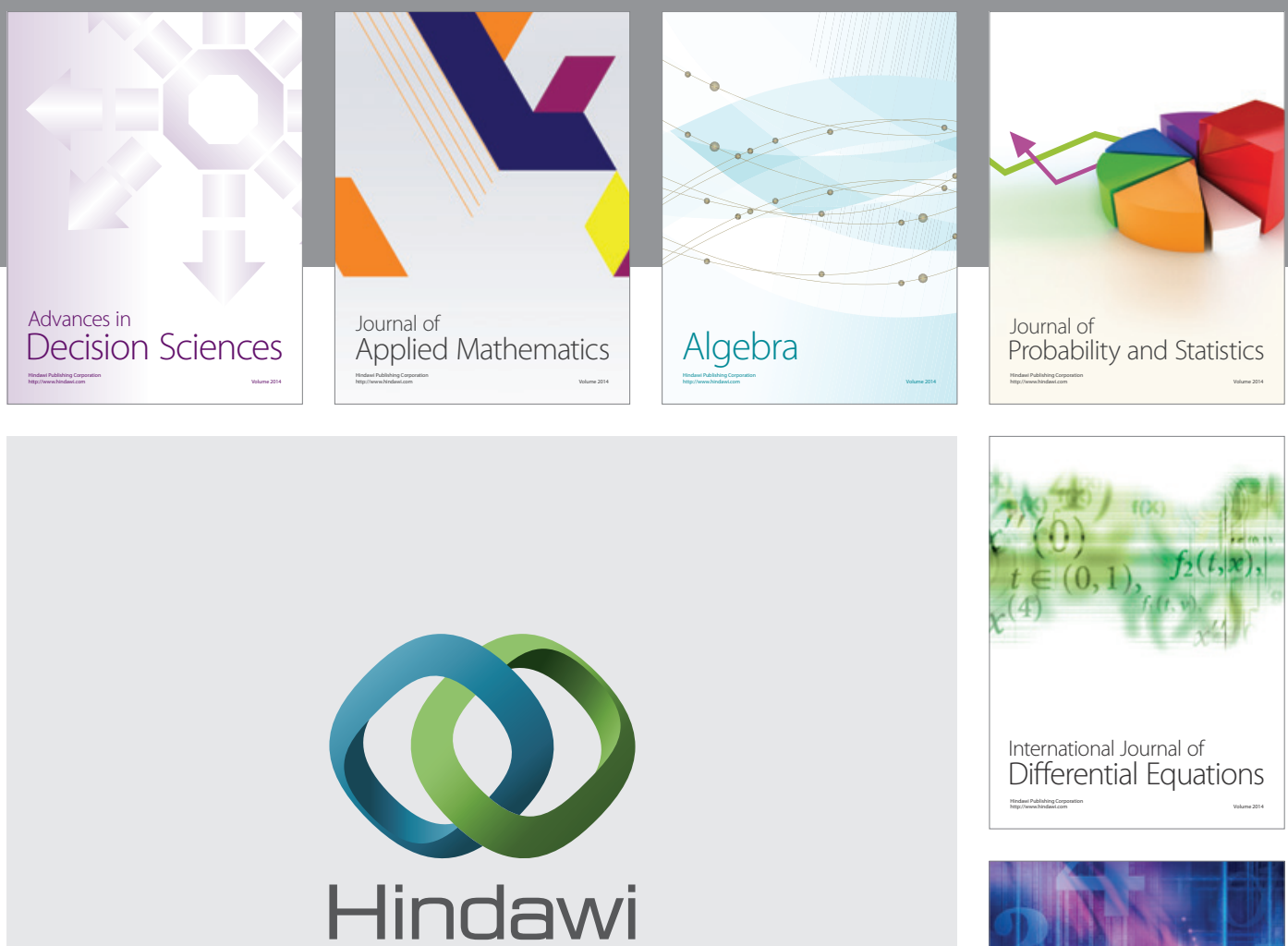

Submit your manuscripts at http://www.hindawi.com
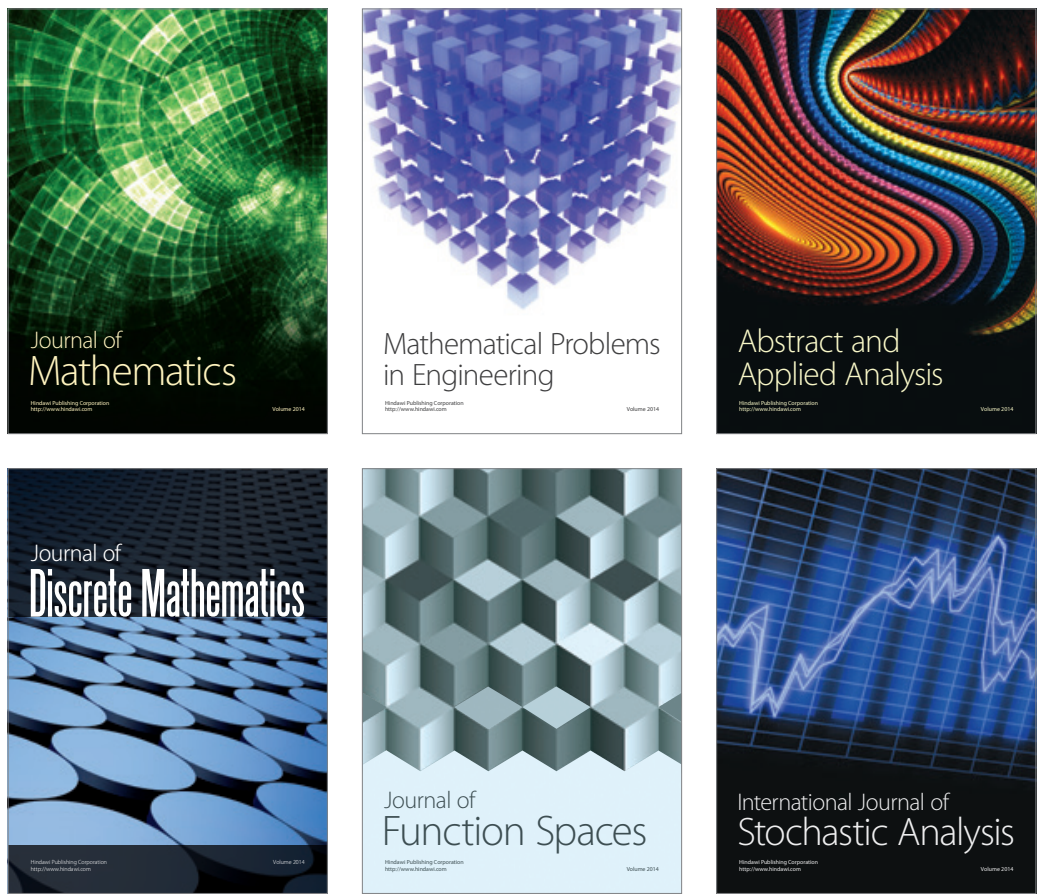

Journal of

Function Spaces

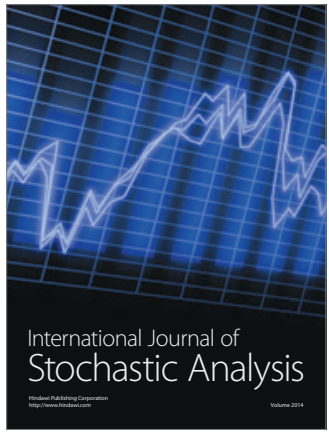

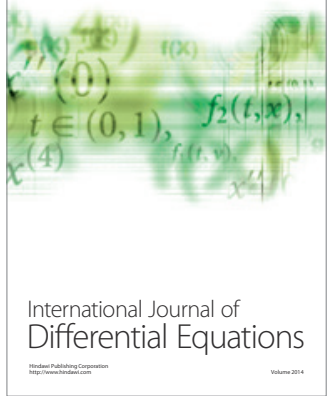
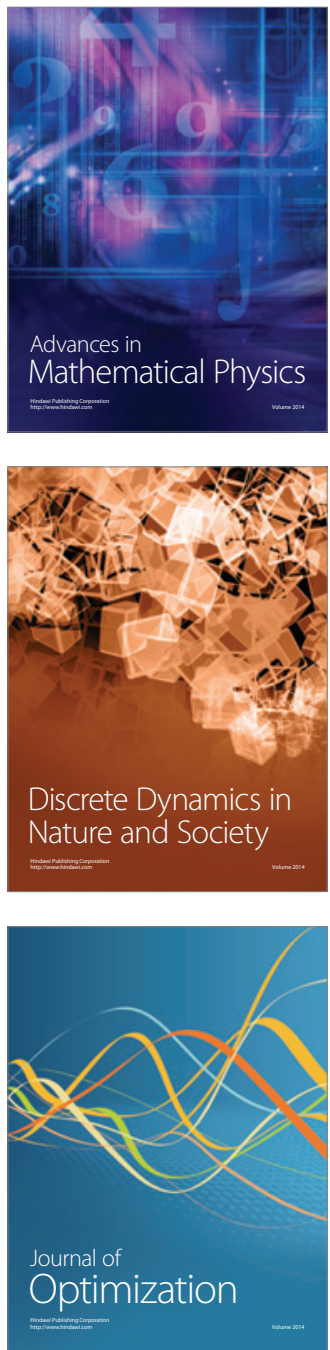